\title{
AffinityTable - A Hybrid Surface for Supporting Affinity Diagramming
}

\author{
Florian Geyer, Ulrike Pfeil, Jochen Budzinski, Anita Höchtl, and Harald Reiterer \\ University of Konstanz, 78457 Konstanz, Germany \\ \{firstname. lastname\} @uni-konstanz.de
}

\begin{abstract}
Using affinity diagramming as an example, we investigate interaction techniques for supporting collaborative design activities. Based on an observational study, we identified design guidelines that need to be addressed to find a close fit to embodied practice. Using this knowledge, we designed and implemented AffinityTable, a hybrid surface for supporting affinity diagramming. The tool combines digital pen \& paper with an interactive table and tangible tokens. An additional vertical display is used to support reflection and group coordination.
\end{abstract}

Keywords: design tools, collaborative design, affinity diagramming, realitybased interaction, digital pen \& paper, hybrid interactive surfaces.

\section{Introduction}

Affinity diagramming is a collaborative design method applied early in the design process for analyzing a design problem or to create first design solutions. It is typically practiced with pen \& paper and a shared surface such as a whiteboard or a large wall. It consists of three main phases: (i) generating (participants individually create content on sticky notes), (ii) sharing (participants present and collect notes), and (iii) structuring (participants arrange notes into meaningful categories). While teams of designers often apply similar techniques within the design process, only few existing digital tools explicitly support these without imposing limitations on the workflow, social coordination and embodiment of thought. Beyer \& Holtzblatt [1] explicitly recommend traditional media for practicing affinity diagramming since using desktop-based digital tools would isolate participants, leading to a breakdown of communication that is vital for a shared understanding in the group. However, in this paper we argue that emerging technologies, like digital pen \& paper, large highresolution displays, multi-touch and tangible interaction, can be utilized to overcome these limitations and may offer additional benefits that go beyond what is possible with traditional physical-only tools. Therefore, we contribute AffinityTable, a hybrid tabletop system for supporting affinity diagramming. It allows users to follow their traditional workflow and embodied practice by still augmenting typical tasks in a lightweight way. Furthermore, it preserves the benefits of physical material during divergent idea generation activities, while enhancing convergent activities through artifact handling support and by providing focus and context views. 
Our work extends related research in computing environments for co-located design work. The Designer's Outpost [2] introduced paper-based interaction concepts in the context of collaborative web site design. It combines physical sticky notes with an interactive whiteboard system. By using overhead image capture, notes can be combined with digital ink annotations. The Shared Design Space [3] combines digital pen $\&$ paper with projected images on a pen-operated interactive table for highresolution drawing with multiple users. Optical marker tracking is utilized to recognize the location of paper on the table. Pictionaire [4] uses overhead image capture and projection to combine physical artifacts with digital annotations for collaborative design work on a hybrid tabletop system. BrainStorm [5] combines multiple pen-operated displays to support brainstorming processes. Digital notes are created on an interactive table and transferred to a wall projection for discussion and clustering. The Designer's Environment [6] supports the KJ creativity method with multimodal interactions for grouping and linking of digital notes on a tabletop system. Handwritten notes are sent to the table from tablet PCs. Text recognition is used on the tablets for retrieving images from the web according to the content of notes.

Our approach extends these efforts with hybrid interaction techniques that make use of both physical and digital representations to better integrate with the social and embodied characteristics of existing workflows. Hybrid surfaces are proposed in order to explore the boundaries between direct touch interfaces and tangible elements [7]. In the following, we describe design guidelines that we derived from an observational study before presenting our proposed workspace design and interaction techniques.

\section{Design Guidelines}

One of the main ideas of reality-based interaction [8] is to build upon characteristics of reality to make interfaces more natural. Jacob et al. recommend looking at reality first to identify critical issues. Computational power may then be used to address those issues that do not work well and can be improved by using technology. However, mechanisms that work good in reality should be preserved. We propose to use this understanding for mapping our potential design space. Therefore, we conducted an observational study of students practicing affinity diagramming. The goal was to identify issues that are critical to the success of the technique. These findings are to be used as a foundation for the design of adequate hybrid interaction techniques that combine physical and digital representations.

We observed three groups of students within a practical session in context of an interaction design course. The group sessions were videotaped and lasted between 1.5 and 2 hours. In addition, each group session was observed by a researcher who made notes of the group activities. Based on the procedures of qualitative content analysis, separate categories were inductively developed for following aspects: interaction with physical artifacts, use of different workspaces and attention and coordination. In the following, we will only describe the most important findings that led to the design of AffinityTable and relate them to supporting results from other sources. By relating our findings to that of other researches, we can improve validity of our results and may infer specific guidelines for the design of an adequate digital workspace. 

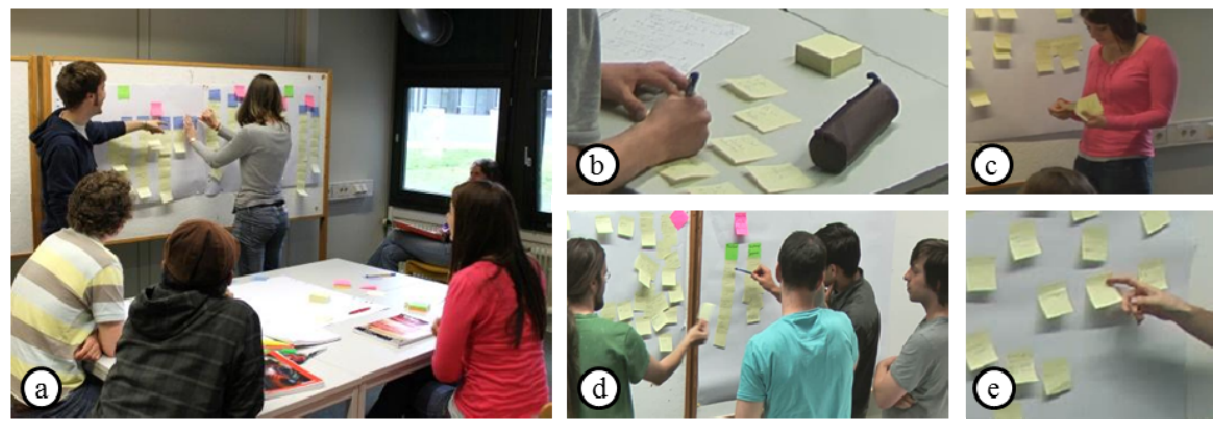

Fig. 1. An observation revealed important characteristics of embodied practice

Based on our analysis we found that paper artifacts are especially useful during divergent activities, like individual ideation, where a large number of different artifacts are created in rapid cycles. Participants also used paper notes for individual reflection, like flipping through created content to search for inspiration. This is in line with results from a study reported by Cook \& Bailey [9] on the use of paper in professional design practice. The authors conclude that paper is highly appreciated due to its unique affordances that support both fluent access and social interactions. Klemmer et al. [2] also emphasize the benefits of paper material for informal design activities. Therefore, we conclude that paper may be used to support individual ideation and reflection activities.

The table was mainly used for individual work. Personal workspaces (see Fig. 1, b) were used for reflection and for preparing content for presentation in the group. However, collaborative work like presenting and discussing took place at the whiteboard. This finding is supported by Vyas [10], who observed the appropriation of physical surfaces in design studios. The author reports that horizontal surfaces mainly serve as a space for action, while vertical surfaces are useful for reflection during convergent activities. We argue that this is due to the differences in accessibility of artifacts and display space. However, we observed that when engaging in actions during convergent phases, the vertical display was frequently blocked by some members of the group (see Fig. 1, a,d), thereby limiting access and visibility to the other participants, which may lead to communication problems. This issue however correlates with the number of participants. Yet, the transfer of notes between personal and shared workspace, which was required for presentation, was accompanied with physical restrictions (see Fig. 1, c) due to the distance between these work surfaces. Like other researchers [5,6], we also consider personal workspaces as essential for the efficiency of creative group work as they moderate social factors like evaluation apprehension by providing a semi-private retreat from the group. Other social factors like production blocking and free riding are partly addressed by the design technique itself. Therefore, the basic workflow of a design technique should not be changed.

During phases of discussion, we observed rapid changes of attention between personal and shared workspaces and between detailed discussions of specific artifacts (see Fig. 1, e) and coordination tasks that required an overview over all artifacts. 
However, we also found that this practice may lead to coordination and communication issues when dealing with a large number of artifacts during discussion. At some points, participants had problems following discussion due to rapid shifts of attention or because some artifacts on the shared work surface were blocked by other participants.

We therefore summarize our findings with following design guidelines: support individual ideation and reflection with paper (DG1); support personal workspaces (DG2); provide support for switching between action and reflection (DG3); ease the transfer of artifacts between workspaces (DG4); preserve the workflow of the original technique (DG5).

\section{A Hybrid Surface for Supporting Affinity Diagramming}

AffinityTable was designed in an iterative design process. In a first step, a workspace was developed to address the findings from our study. In a second step, specific hybrid interaction techniques were designed to support and augment the workflow and tasks within affinity diagramming.
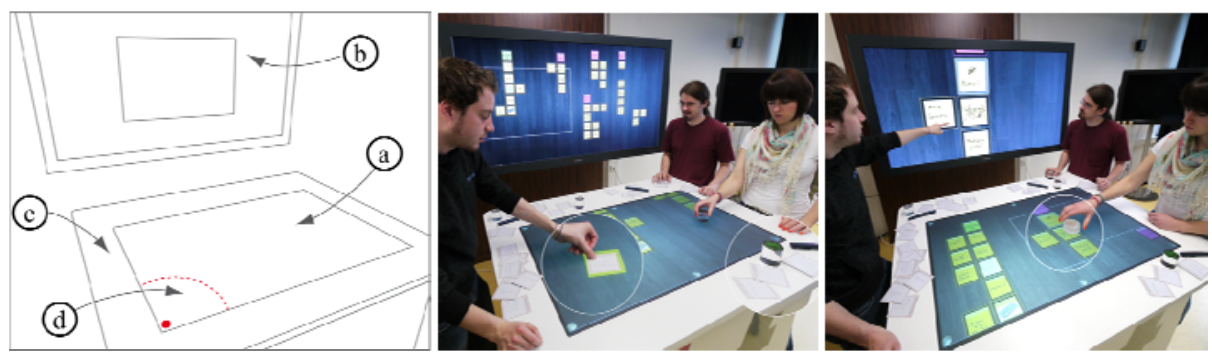

Fig. 2. AffinityTable combines different workspaces by using digital pen \& paper, an interactive table with tangible tools and a coupled, high-resolution vertical display

\subsection{Workspace Design}

Our goal when designing AffinityTable was to replicate the benefits of reality and to improve identified issues by using interactive technology. The physical workspace used in practice was composed of one vertical and one horizontal work surface (see Fig. 1, a). We found that these work surfaces are important for preserving the workflow of the technique. Therefore, we decided to keep the basic layout, but to provide smoother transitions between action and a reflection by the means of a virtual workspace across two interactive displays (DG3). Fig. 2 shows the final workspace design, which is based on an interactive table for collaboratively interacting and reflecting on a focused region of the workspace (shared action space, see Fig. 2, a) and a high-resolution wall display (process reflection space, see Fig. 2, b) for reflecting on the overall progress of the process. Thereby, the table displays a magnified region of the whole workspace, which is shown on the wall display. The workspace can be navigated on the table with panning gestures. A focus box on the 
vertical display visualizes the region that is currently displayed on the table. Digital pen \& paper is used on the non-interactive rim of the table for supporting individual ideation and reflection (personal spaces, see Fig. 2, c). Personalized interactive areas at the corners of the table (transfer spaces, see Fig. 2, d) provide a zone for additional individual actions. The coupled vertical display can also be used for dynamic views on selected artifacts during phases of discussion (see Fig. 2, right).

\subsection{Interaction Techniques}

AffinityTable supports the workflow of affinity diagramming with hybrid interaction techniques for copying, clustering, piling, and collecting. Additional functionalities that augment physical practice are integrated with interaction techniques for highlighting, focusing, searching, and image retrieval. We aimed at incorporating tangible elements for improving visibility of actions and group awareness.

Copying. In physical practice, notes are created and reflected individually in personal workspaces. AffinityTable preserves this ability by providing small paper notes and digital pens. Handwriting and sketches are instantly transferred to the system via a wireless connection. Physical notes can be copied to the shared workspace by placing them on the interactive table (see Fig. 2, center). A digital copy of the physical note appears within the virtual workspace, while the physical copy remains in the personal workspace. This technique allows sharing and presenting of artifacts without physical restrictions while still preserving the ability to reflect upon all individual contents in the personal workspace throughout the process (DG1,DG2). It also enables rapid transfer between the personal and the shared workspace (DG3,DG4).
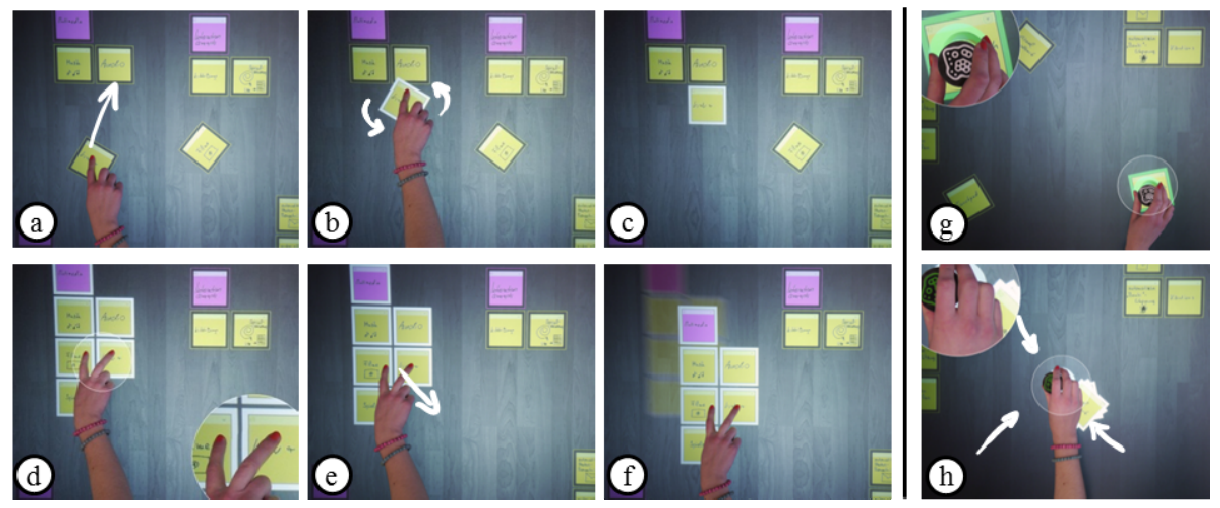

Fig. 3. Clustering notes with touch manipulations (a,b,c); moving clusters of notes by using multiple fingers $(\mathrm{d}, \mathrm{e}, \mathrm{f})$; collecting notes by using colored tokens $(\mathrm{g}, \mathrm{h})$

Clustering and Piling. During convergent activities, notes are discussed and organized into meaningful arrangements. Once copied into the shared workspace, digital notes can be dragged, rotated and flicked by using multi-touch manipulations on the interactive table. It is also possible to change the color of notes or to delete 
notes, which are basic requirements for clustering. To facilitate the collaborative organization of notes into groups, we included a simple clustering algorithm that automatically aligns and associates notes when released close to each other (see Fig. 3 , a,b,c). Piles of notes can be created by releasing notes on top other notes. Clusters and piles can be moved by dragging them with multiple fingers (see Fig. 3, d,e,f). These interaction techniques closely resemble collaborative sorting actions at the whiteboard (DG5). However, since all user action is focused on a horizontal surface, which is accessible to all participants, this might lead to more equal access and increased awareness of actions within the group.

Collecting. When collecting notes or clusters from arbitrary locations within the shared workspace, it is a tedious task to move them individually via touch manipulations across longer distances in the virtual workspace. In physical practice, notes can be collected into mobile piles (see Fig. 1, c) and thus can be easily transferred to remote locations. AffinityTable replicates this ability by providing multiple two-sided tokens. Users can select digital notes by using the colored side of the tokens (see Fig. 3, g). The notes are then marked in that color. By turning over the tokens and placing them on the table with the blank side, selected notes are moved to this new location (see Fig. 3, h). Because multiple tokens can be used at the same time, this technique also enables loosely coupled parallel work. The tangibility of the tokens and the colored marking used as a proxy for mobile piles may increase the visibility of these parallel actions.

Highlighting and Focusing. During discussion, deictic references on notes are often used as a form of communication and for coordination in the group (see Fig. 1, d,e). AffinityTable augments these interactions by providing additional functionality. When touching digital notes, they are highlighted around their border with a glowing effect. Each highlight fades out after five seconds. When clustering notes, the fading glow implicitly communicates a history of actions. In addition, a token can be used to focus on a specific region within the workspace during reflection (DG3). By turning the token (see Fig. 4, a), the users can change the zoom-factor of the magnified region displayed on the vertical display (see Fig. 2, right). We chose a single tangible element as focusing tool because we believe its limited accessibility may stimulate negotiations about the focus of discussion.
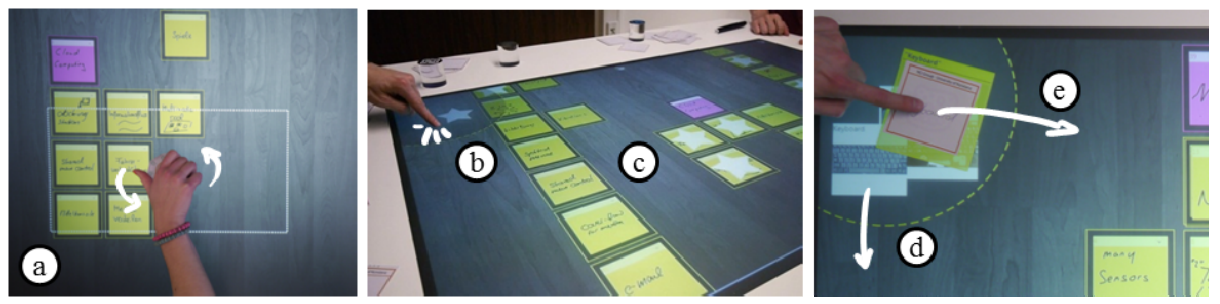

Fig. 4. Focusing on a specific region in the workspace (a); searching for notes (b,c); retrieving and adding images based on the content of notes $(\mathrm{d}, \mathrm{e})$. 
Searching. AffinityTable provides a lightweight search function based on the author of notes. By tapping personal symbols that are available in the transfer spaces at the corners of the table (see Fig. 4, b), the notes created by the corresponding user are marked with that symbol (see Fig. 4, c). This allows participants to look up the responsible author of a particular issue or idea during discussion. It also allows reflecting on participants contributions to certain clusters and therefore may provide information about consensus in the group (DG3). This search function may introduce positive effects by reducing free riding, but may also have negative effects by increasing evaluation apprehension. However, since notes are anonymous throughout the session and identity is only revealed on demand, this technique may stimulate individual contributions without putting too much pressure on participants.

Image Retrieval. When presenting ideas and issues it can be helpful to add additional information to make a clear point. Therefore, AffinityTable provides image retrieval based on the content of notes. When placing notes into transfer spaces at the corners of the table, five related images from the web are displayed around the physical note. Users may then drag digital images along with a copy of the physical note to the shared workspace (see Fig. 4, d,e). Images in the workspace can be clustered along with digital notes. Hence, they can also be used to visually point out important clusters by adding representative images. This technique may have positive influence on fixation effects because the selection of images can be used to further open up divergent activities. Instead of using images from the web, this technique may also be used to augment notes with other data from custom design knowledge repositories.

\subsection{Implementation}

AffinityTable runs on a custom-built interactive table that measures $128 \mathrm{~cm} \times 157 \mathrm{~cm} \mathrm{x}$ $105 \mathrm{~cm}$. The non-interactive rim $(20 \mathrm{~cm})$ was designed for supporting leaning, personal workspaces and for avoiding involuntary interaction. The table provides adequate space for up to five users. The graphics are rear-projected with a WUXGA projector (1900px x 1200px). Multi-touch and object recognition is provided with IR illumination and three tiled XGA cameras (1024px x 768px). The vertical display measures $76 \mathrm{~cm} \times 162 \mathrm{~cm}$ and features $4 \mathrm{~K}$ resolution (4096px x 2160px). The software runs on two networked workstations with MS Windows 7. The interface is implemented using the ZOIL framework [11] that integrates WPF multi-touch controls with distributed user interface synchronization and zooming functionality. We use ReacTIVision ${ }^{1}$ for marker tracking and Squidy [12] for finger tracking. Data from Anoto's ADP-301 digital pens is received via Bluetooth by using the Anoto streaming $\mathrm{API}^{2}$. Text recognition is implemented using MS Windows 7 SDK. We use the Yahoo image search service for retrieving digital images.

\section{Conclusion}

In this paper we presented AffinityTable, a hybrid surface for supporting affinity diagramming. Based on an observational study, we identified design guidelines for a close fit to embodied practice. This knowledge was used for the design of a

\footnotetext{
${ }^{1}$ http: //reactivision. sourceforge.net

${ }^{2}$ http: / / www . anoto.com
} 
workspace that combines digital pen $\&$ paper with an interactive table and tangible tools for supporting the basic workflow of the design technique and for providing additional functionalities. A vertical display is used for supporting reflection-in-action and for enhancing discussion and coordination. The described interaction techniques can be adapted for similar design techniques that share the phases of idea-generation, idea-presentation and idea-discussion. However, this paper does not provide empirical evidence on the positive effects of the proposed interaction techniques. In future work we will improve AffinityTable by adding more powerful clustering techniques. Eventually, a user study is necessary to gain a deeper understanding of the tool's effects on the process of the design technique, efficiency and social factors.

Acknowledgements. This research is funded by the German Research Foundation DFG (Deutsche Forschungsgemeinschaft) under grant number RE 1843/3-1.

\section{References}

1. Beyer, H., Holtzblatt, K.: Contextual design: defining customer-centered systems. Morgan Kaufmann, San Francisco (1998)

2. Klemmer, S.R., Newman, M.W., Farrell, R., Bilezikjian, M., Landay, J.A.: The Designers' Outpost: A tangible interface for collaborative web site design. In: Proceedings of UIST 2001, pp. 1-10. ACM Press, New York (2001)

3. Haller, M., Leithinger, D., Leitner, J., Seifried, T., Brandl, P., Zauner, J., Billinghurst, M.: Shared design space: sketching ideas using digital pens and a large augmented tabletop setup. In: Advances in Artificial Reality and Tele-Existence, pp. 185-196. Springer, Heidelberg (2006)

4. Hartmann, B., Morris, M.R., Benko, H., Wilson, A.D.: Pictionaire: supporting collaborative design work by integrating physical and digital artifacts. In: Proceedings of CSCW 2010, pp. 421-424. ACM Press, New York (2010)

5. Hilliges, O., Terrenghi, L., Boring, S., Kim, D., Ritcher, H., Butz, A.: Designing for collaborative creative problem solving. In: Proceedings of C\&C 2007, pp. 137-146. ACM Press, New York (2007)

6. Tse, E., Greenberg, S., Shen, C., Forlines, C., Kodama, R.: Exploring true multi-user multimodal interaction over a digital table. In: Proceedings of DIS 2008, pp. 109-118. ACM Press, New York (2008)

7. Kirk, D., Sellen, A., Taylor, S., Villar, N., Izadi, S.: Putting the physical into the digital: issues in designing hybrid interactive surfaces. In: Proceedings of BCS HCI 2009, pp. $35-44$ (2009)

8. Jacob, R.J.K., Girouard, A., Hirshfield, L.M., Horn, M.S., Shaer, O., Solovey, E.T., Zigelbaum, J.: Reality-based interaction: a framework for post-WIMP interfaces. In: Proceedings of CHI 2008, pp. 201-210. ACM Press, New York (2008)

9. Cook, D.J., Bailey, B.P.: Designers' se of paper and the implications for informal tools. In: Proceedings of OZCHI 2005. ACM Press, New York (2005)

10. Vyas, D.: Artful surfaces in design practices. In: Proceedings of CHI EA 2009, pp. 2691-2694. ACM Press, New York (2009)

11. Jetter, H.-C., Gerken, J., Zöllner, M., Reiterer, H.: Model-based design and prototyping of interactive spaces for information interaction. In: Proceedings of HCSE 2010, pp. 22-37. Springer, Heidelberg (2010)

12. König, W.A., Rädle, R., Reiterer, H.: Interactive design of multimodal user interfaces reducing technical and visual complexity. Journal on Multimodal User Interfaces 3(3), 197-213 (2010) 